\title{
Extraction and analysis of morphologic and hydrologic properties for Luvuvhu River Catchment in Limpopo province, South Africa
}

\author{
P. M. Kundu, R. L. Singo, J. O. Odiyo, F. I. Mathivha \\ \& R. N. Nkuna \\ Department of Hydrology and Water Resources, \\ University of Venda, South Africa
}

\begin{abstract}
Physiographic and topographic changes in Luvuvhu River Catchment are negatively impacting on the catchment hydrology of the area. A study was carried out to extract and analyze the morphologic and hydrologic properties using GIS techniques. Digital Elevation Modeling hydro-processing procedures were used within an ArcGIS environment where Arc hydro tools were used to extract and show the spatial distribution of the properties. A hydrologically correct Digital Elevation Modeling (DEM) was generated and used to obtain primary and derived terrain elements. The results showed the automated delineation of sub-catchments and the spatial distribution of morphometric and hydrologic properties. The analysis showed how the physiographic changes were impacting on the river regimes and water resources.

Keywords: catchment, Digital Elevation Modeling, GIS techniques, hydrologic properties, morphometric properties.
\end{abstract}

\section{Introduction}

Luvuvhu River catchment exhibits diverse land use and land cover patterns that are influenced by seasonality and socio-cultural practices of the local communities. From 1950, the catchment has been undergoing land cover changes caused by building new urban centres and establishing new villages while expanding old ones, and also clearing forestland for agriculture. In many instances, indigenous forests were cleared and replaced with eucalyptus and pine 
plantations in the highlands [1]. Conversion of natural landscape for agricultural and urban uses often impacts on soil integrity, nutrient fluxes, and native species assemblages [2] and may lead to reduced base flows in the catchment. In this study, extraction and analysis of morphologic and hydrologic properties was carried out using GIS techniques. An analysis of drainage networks from Digital Elevation Models (DEMs) was carried out as a viable approach for possible automated floodplain delineation to estimate flood risks.

\section{The study area}

Luvuvhu River Catchment shown in Figure 1 is located between latitudes $22^{\circ} 17^{\prime} 57^{\prime \prime S}$ and $23^{\circ} 17^{\prime} 31^{\prime \prime S}$ and longitudes $29^{\circ} 49^{\prime} 16^{\prime \prime} \mathrm{E}$ and $31^{\circ} 23^{\prime} 02^{\prime \prime} \mathrm{E}$ in Limpopo province of South Africa. The region covers an area of approximately $5941 \mathrm{~km}^{2}$, situated on a plateau of 1312 meters above sea level [3]. The topography of the catchment is undulating with a few prominent ridges extending in an east-west direction, whereas the monotony of the area is relieved by separated hills and ranges [4].

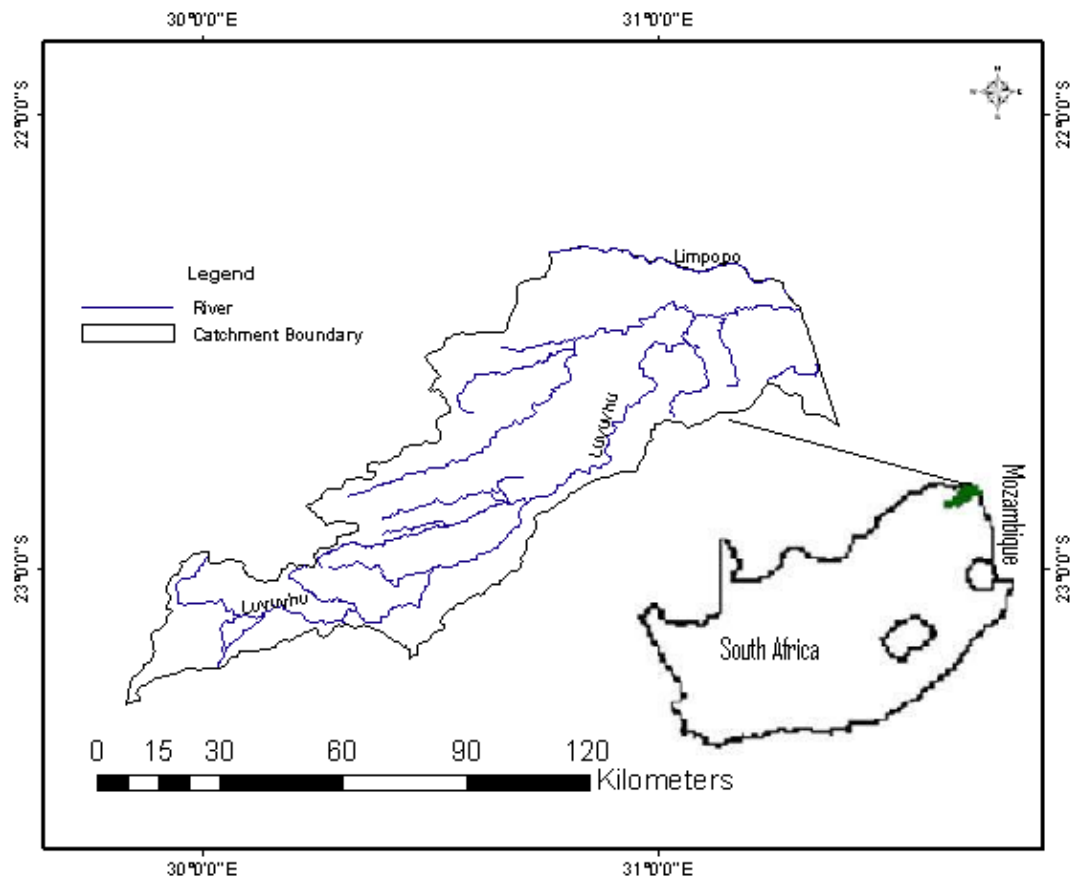

Figure 1: The study area and sub-catchments of the basin. 


\section{Materials and methods}

Automated extraction and spatial analysis of the morphologic and hydrologic properties was carried out. DEM hydro-processing procedures were used within ArcGIS environment where ArcHydro tools were used to extract the measurable attributes.

\subsection{DEM generation}

A hydrologically correct DEM was generated on the basis of contours, rivers and stream lines and spot heights from topographic maps of scale 1:50,000. Since contours formed the source data for modeling, the TOPOGRID algorithm was selected to create DEMs upon which surface features were draped. The resolution of the DEM was set at $50 \mathrm{~m}$ and the land surface characteristics were quantified on a cell by cell basis. A discretized thin-plate spline technique enabled the fitted DEM to show abrupt changes in the terrain such as valleys and ridges.

\subsection{Surface modeling}

Surface modeling was undertaken to translate discrete points into a continuous surface that represented the geographic distribution of topographic features using Fast Fourier Transforms, which computed the transform of the signal $f(x)$ by a series of harmonically related sinusoids. The terrain surface from which morphologic and hydrologic properties were automatically extracted was created using models of the form presented in equations (1) to (8) where the variance of a continuous sampled signal $\left(\sigma_{s}\right)$ was computed as

$$
\sigma_{s}^{2}=2 \sum_{k=1}^{N / 2}\left\{1-H\left(v_{k}\right)\right\}^{2}\left|F\left(v_{k}\right)\right|^{2}
$$

where

$$
\mid F\left(\left.v_{k}\right|^{2}=|F(k)|^{2}=R F(k)^{2}+L F(k)^{2}, v_{k}=k v_{1}=\frac{k}{L}\right.
$$

was the amplitude spectrum of the output $f^{\prime}(x)$

and $H\left(v_{k}\right)=0$ for $v_{k}>\frac{1}{2 \Delta x}$

was generalized as

$$
e(x, y)=f(x, y)-\left(f^{\prime} x, y\right)
$$

where 


$$
f(x, y)=\sum_{k 1=0}^{k 1} \sum_{k 2=0}^{k 2} a_{k 1 k 2} \sin \left(2 \pi v_{k 1} x+2 \pi u_{k 2} y+\varphi_{k 1 k 2}\right)
$$

with several intervals $\Delta x_{1}=\Delta y_{1}$ by using bilinear interpolation.

$$
\sigma^{2}=\frac{1}{L X L Y} \int_{0}^{L Y L X} \int_{0}^{2} e^{2}(x, y) d x d y
$$

If $\quad L X, L Y \rightarrow \infty$,

$$
\sigma^{2}=\int_{-\infty}^{\infty} \int|E(v, u)|^{2} d v d u
$$

where

$$
\begin{gathered}
E(v, u)=F[e(x, y)], \sigma^{2}=\sigma_{s}^{2}+\sigma_{R}^{2}, \\
\sigma_{s}^{2}=\sum_{k 1=-\frac{N 1}{2}}^{\frac{N 1}{2}-1} \sum_{k 2=\frac{-N 2}{2}}^{\frac{N 2}{2}-1}\left\{1-H\left(v_{k 1}, u_{k 2}\right)\right\}^{2}\left|F\left(v_{k 1}, u_{k 2}\right)^{2}\right|
\end{gathered}
$$

$H(v, u)$ being zero for $v>\frac{1}{2 \Delta x}$ or $u>\frac{1}{2 \Delta y}$

$$
F\left(v_{k 1}, u_{k 2}\right)=F(k 1, k 2)=\frac{1}{N 1 N 2} \sum_{L 1=0}^{N 1-1 N 22-1} \sum_{L 2=0} f(L 1, L 2) e^{-j 2 \pi\left(\frac{L 1 k 1}{N 1}+\frac{L 2 k 2}{N 2}\right)}
$$

where $\mathrm{N} 1$ was the number of sampled points in the $x$-direction and $\mathrm{N} 2$ was the number of points in the $y$-direction.

Taking the circumference of the Earth to be about $40,000 \mathrm{~km}$, the maximum spherical harmonic degree $l_{\max }$ would be at least $10^{4}$ and thus $10^{8}$ coefficients were needed. But this was impractical from a computational standpoint since most of the surface would have the same depth, making most of the coefficients to contain information that is not useful. Since the sampling was based on a grid of about $100 \mathrm{~m}$, a smaller patch of dimensions $100 \mathrm{~m}$ by $100 \mathrm{~m}$ was used to generate a fourier representation of only $10^{4}$ coefficients.

\subsection{Extraction of morphologic and hydrologic properties}

The deterministic eight neighbour (D8) algorithm $[5,6]$ was used to extract the morphologic and hydrologic properties. It was the simplest flow derivation algorithm available for use with grid DEMs and was able to rapidly extract stream networks in a straightforward and consistent manner. Flow was computed within a $3 \times 3$ cell kernel, with the central cell only knowing about its 
surrounding eight cells. This technique formed the basis of hydrological networks derived from DEMs due to its robust nature and rapid straightforward implementation.

The measurable terrain and morphometric attributes shown in Table 1 were derived based on quadratic computations as shown in equation 9:

$$
h=A f^{2} g^{2}+B f^{2} g+C f g^{2}+D f^{2}+E g^{2}+F f g+G f+H g+I
$$

where $f$ and $g$ are spatial coordinates and $h$ is elevation. The nine parameters, $A$, $B, C, D, E, F, G, H$ and $I$ were determined from the nine elevations on the $3 \times 3$ window using Lagrange polynomials as described by Zevenbergen and Thorne [7].

Table 1: Derivation of primary and secondary attributes.

\begin{tabular}{|c|c|c|}
\hline Attribute & Category & Calculation method \\
\hline Elevation (h) & TIN & DEM \\
\hline Slope $(\beta)$ & Primary & $\arctan \left\lfloor\sqrt{ }\left(G^{2+H^{2}}\right)\right\rfloor$ \\
\hline Aspect $(\varphi)$ & Primary & $180-\arctan \left[\frac{H}{G}\right]+90 \frac{G}{\mid G}$ \\
\hline Plan curvature $(\Phi)$ & Primary & $2 \frac{D G^{2}+E H^{2}-F G H}{G^{2}+H^{2}}$ \\
\hline Profile curvature $(\hat{\mathrm{w}})$ & Primary & $2 \frac{D H^{2}+E G^{2}-F G H}{G^{2}+H^{2}}$ \\
\hline Curvature $(\chi)$ & Primary & $2 E+2 D$ \\
\hline Contributing area $(A i)$ & Primary & $\frac{1}{b} \sum_{i=1}^{n} a_{i}$ \\
\hline \multicolumn{3}{|l|}{ Compound Topographic } \\
\hline Index (CTI) & Secondary & $\ln \left[\frac{A_{j}}{\tan \beta}\right.$ \\
\hline Stream Power Index (SPI) & Secondary & $A_{j} \tan \beta$ \\
\hline Slope Aspect Index (SAI) & Secondary & $\varphi \tan \beta$ \\
\hline
\end{tabular}




\subsection{Automated catchment delineation}

A DEM was smoothed prior to analysis to reduce the size and number of sinks using the fill sinks tool. The resulting surface was used to define the surface drainage pattern. Flow direction and flow accumulation layers were then calculated. The stream grid was then segmented into sections representing headwater tributaries or segments between confluences, with each segment assigned a unique grid code identifier. The resulting link grid was used to generate a catchment grid based on the values held by each stream segment. These catchment grids were converted to polygon vector features, with single cell catchments automatically dissolved. Stream layers were created using flow direction and pour points as input layers resulting in identification of flood inundation areas. Figure 2 is a schematic representation of the DEMhydroprocessing method adopted in this study.

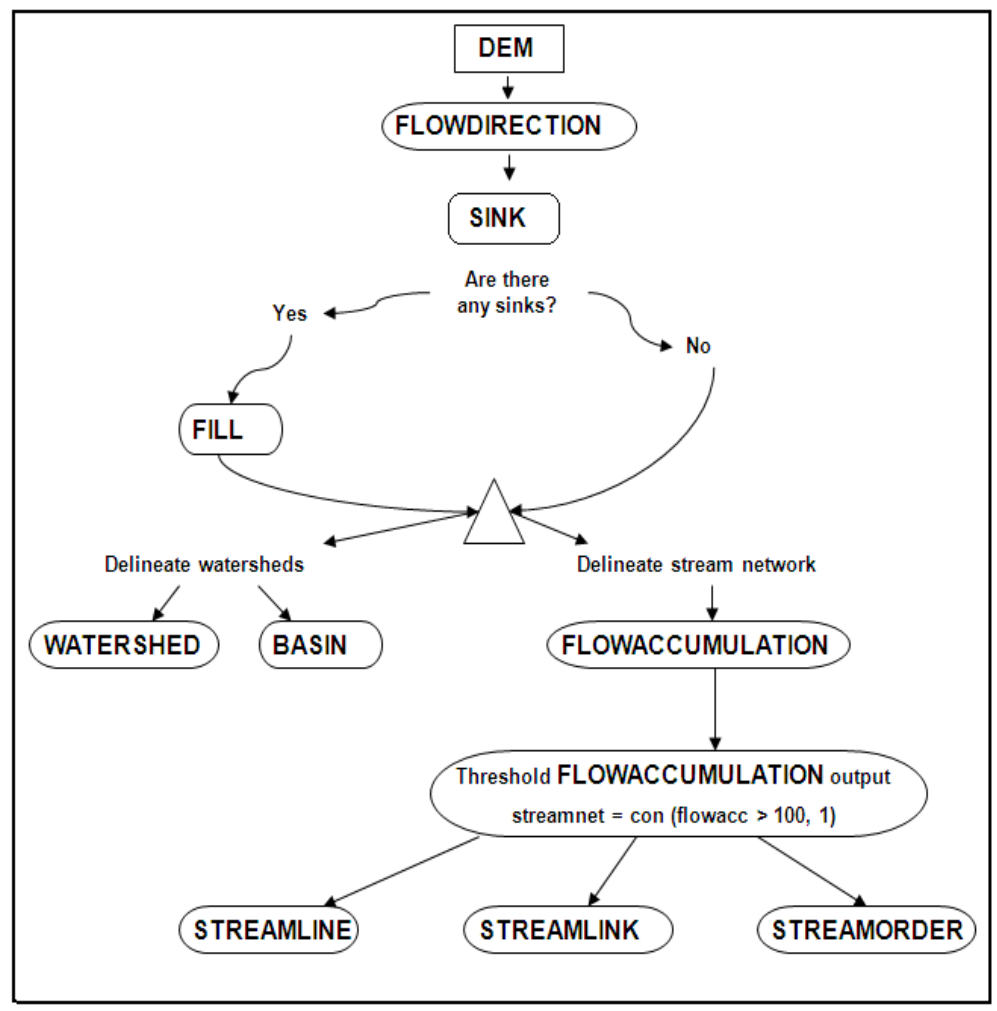

Figure 2: Schematic representation used in creating a hydrologically correct DEM. 


\section{Results and discussion}

Morphologic parameters were divided into areal, relief and network properties. Figure 3 shows the hydrologically correct DEM that was generated and used as a source of height data for modeling altitude-correlated properties. Since contour lines were used for DEM creation, they largely influenced the final product due to data imperfections created during data capture.

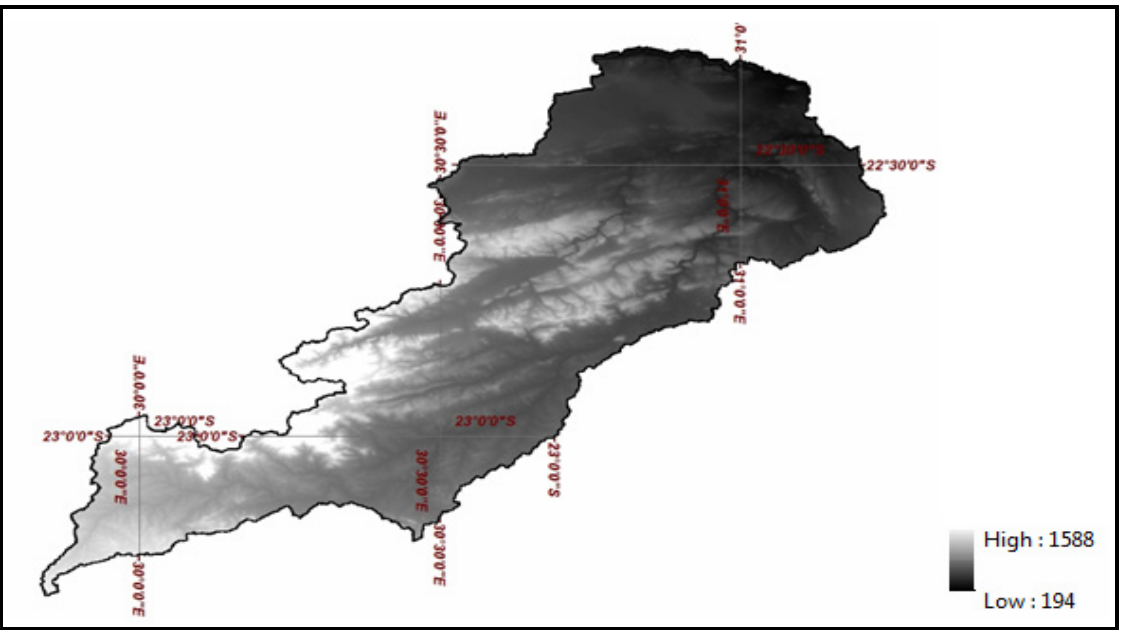

Figure 3: Hydrologically correct DEM for Luvhvu River catchment.

Assessment of data quality involved extracting frequency histograms of elevations, and Figure 4 shows the frequency distribution for the DEM, which indicated that the DEM had a representative number of pixels with constant $\mathrm{Z}$ values, and did not therefore have a large number of flat regions dominating. Imperfections contained in the DEM could directly compromise the results of analysis for hydrological modeling and should therefore be as little as possible.

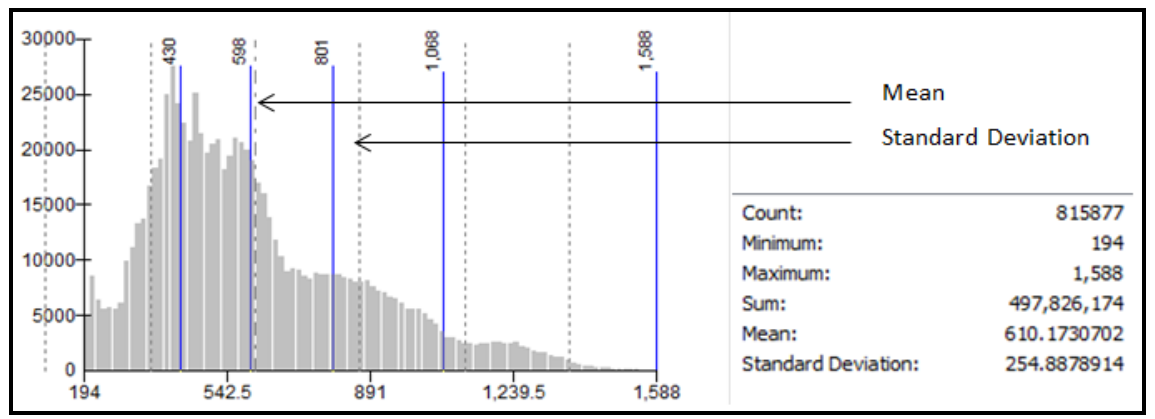

Figure 4: Frequency distribution of heights in the Luvhvu River catchment. 
Figure 5 shows sub-catchment areas and their topographic attributes including shape and length needed for all physically based hydrological models. Features such as terrain slope, drainage network, and catchment boundaries are essential input parameters for distributed catchment modeling. Mapping their spatial distribution is crucial to ensure that human activities do not inhibit the flow of water to natural systems and hence sustain biodiversity at the smallest sub-catchment unit. Based on this, the technique could be used for hydrological analysis of similar catchments and thereby open the planning process to the full advantages of GIS technology. Automated catchment delineation makes it possible for more responsive water resources management programs to be taken at local micro scales.

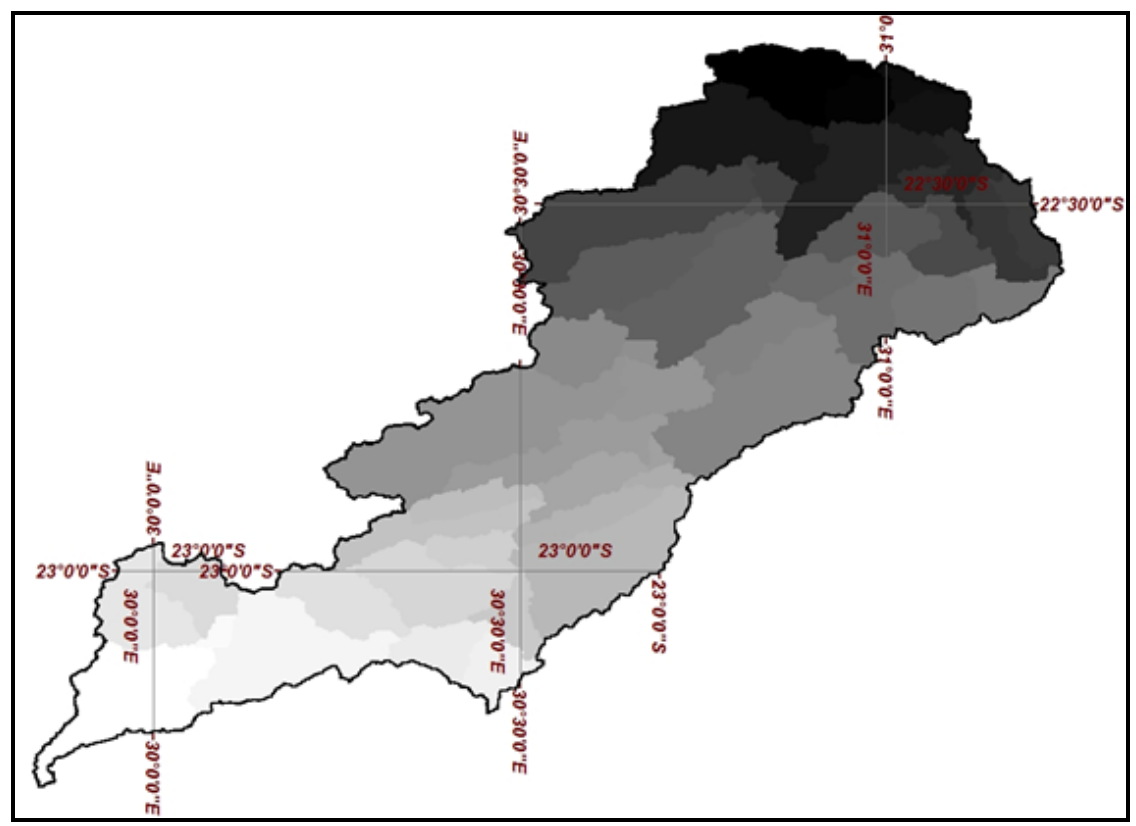

Figure 5: Automated catchment delineation for Luvhvu River catchment.

The spatial distribution of local slopes for Luvuvhu River catchment affected the overall rate of downslope movement for processes such as runoff, erosion hazard, moisture balance and landslide hazards. Extraction of this property is therefore important as it allows overland and near-surface water flow to be modeled using DEMs if surface topography is assumed to be the sole factor in the distribution of water. This helps check the processes of acceleration and deceleration and reduce the effects of erosion and deposition in the catchment.

The flow direction model showed the direction of the steepest downslope neighbour for each cell. The model was similar to the slope direction and was in conformity with the fact that water takes the shortest path to find its own level. The pour points represented the locations where water would most likely be 
found throughout the year even during droughts. Such points would be the best sites for developing community water supply projects as they would serve as reliable sources of water to ensure availability throughout the year. The flow accumulation layer added up all the upstream water available for runoff using the flow direction information layer along the steepest slope. Thus, the water accumulated along the flow paths while being directed by topography.

Knowledge of the compound topographic index (CTI) as a derived attribute was important as it provided the wetness index which could be used to predict zones of saturation in catchments whereas the stream power index (SPI) provided a measure of the erosive power of overland flow. The dominance of aspect, elevation and the slope aspect index (SAI) reflected the fact that the surface horizon was deepest on the plateau followed by the escarpment and plains. The thinnest surface horizons occurred on the ridges and hills. This information is vital for managing farmland in the hilly areas within the catchment such as Piesanghoek macadamia cropland and Tsianda mango farms.

The extracted drainage pattern shown in Figure 6 was of the parallel type. The headwater streams were extracted to the Strahler stream order 3 which allows a stream to increase in order only when two streams of the same order intersect. The drainage network was generated using a constant-threshold method based on the evolution mechanism of River Luvhvu and landform characteristics of the catchment. The characteristics of the extracted networks showed the hillslope travel distance and network link lengths. These properties are important in catchment management as it influences the effects of channel morphology on water quality through the unit stream power. In models of landscape evolution and river incision, unit stream power is extensively used because it evolves dimensional downstream profile of the river channel, and could also be used in river channel migration and sediment transports analysis.

These streams require the near-stream vegetation for environmental flow. When vegetation is removed along stream banks, water temperature increases. This brings about a shift in the micro-ecological structure that may result in resident species being replaced by less desirable ones that are more tolerant to increased temperatures. Sediments also reduce the structural complexity and productivity of aquatic life. The channel lengths estimated the streams following twists and turns as closely as possible. Since the upstream end of the streams are often incorrectly shown on maps, this approach was appropriate and could be used to measure channel lengths from a stream's mouth in an upstream direction. In general, the actual length of the stream is usually underestimated from maps, because twists and turns of the channel are increasingly smoothed at smaller map scales. This model provided the advantage of calculating the length of the upstream and downstream flow path from each cell.

In this study, the average travel distance slope was taken as the average of the slope from each point in the sub-catchment to the next adjacent downslope channel. Adjacent catchments were identified in order to associate topologic information for upstream and downstream connections. In this way, the lower order catchments were merged to a higher order, which was useful for basin scaling. The flow length model was used to estimate the length of the longest 
flow path within Luvuvhu River catchment, which could be used for inundation mapping and floodplain delineation. The longest flow path data could also be used in the rainfall-runoff analysis to determine the time of concentration value necessary for computing surface runoff.

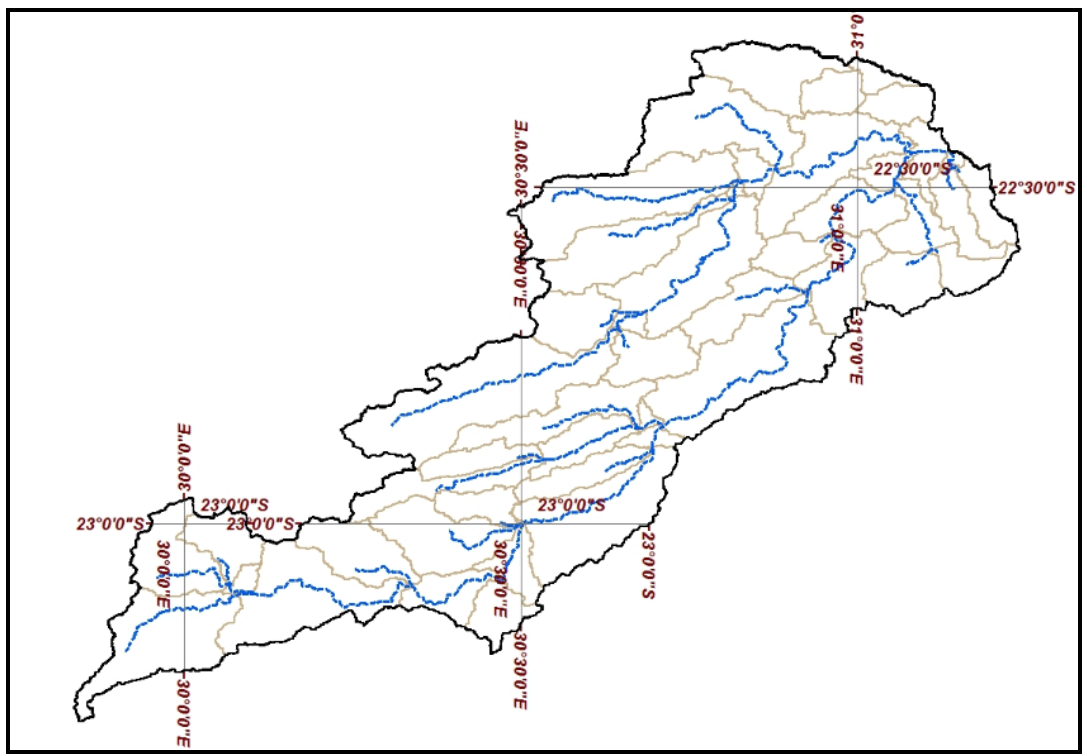

Figure 6: Automated extraction of the drainage network for Luvhvu River catchment.

\section{Conclusion and recommendations}

The use of DEMs was found to efficiently represent ground surface and allow automated extraction of features, thus bringing advantages in terms of processing efficiency, cost effectiveness, and accuracy assessments. The characteristics of the extracted network showed the hillslope travel distance and network link lengths. The effect of channel morphology on water quality could be analysed in more detail since the ability of a stream to transport sediment appeared to be directly related to the rate of energy expenditure as it flowed from higher to lower elevations. The approach provided scope for modeling the storage of water and sediment transport for Luvuvhu River. This technique could therefore be adopted to improve land use planning, water management, communication and telecommunication and extended to all projects which take slopes and elevations in consideration for their functional, structural and aesthetic requirements. 


\section{Acknowledgements}

The authors would like to acknowledge the support of the South African Research Foundation (NRF) and the University of Venta for funding the study. We are grateful to relevant authorities at the Department of Water Affairs (DWA) for providing valuable data historical data.

\section{References}

[1] Van der Waal, B.C.W., 1997. Fundudzi, a unique, sacred and unknown South African lake. South African Journal of Aquatic Science, 23, 42-45.

[2] Powers, J.S., Read, J.M., Denslow, J.S., Guzman, S.M., 2004. Estimating soil carbon fluxes following land-cover change: A test of some critical assumptions for a region in Costa Rica. Global Change Biology, 10, 170181.

[3] DWAF, 2002. A proposed National Water Resources Strategy for South Africa. Proposed First Edition, Department of Water Affairs and Forestry, South Africa.

[4] DWAF, 2004. Directorate: National Water Resource Planning. Internal strategic perspective Luvuvhu/Letaba Water Management Areas. Department of Water Affairs and Forestry, Pretoria, Report No. PWMA 01/000/00/0304.

[5] O'Callaghan, J. F. and Mark, D. M. 1984. The Extraction of Drainage Networks from Digital Elevation Data. Computer Vision, Graphics, and Image Processing, 28:323-344.

[6] Martz, L. W., Garbrecht, J., 1992. Numerical Definition of Drainage Network and Subcatchment Areas from Digital Elevation Models. Computers and Geosciences, 18(6):747-761.

[7] Zevenbergen, L.W., Thorne, C.R., 1987. Quantitative analysis of land surface topography. Earth Surface Processes and Landforms 12, 47-56. 